\title{
FINITE ELEMENT PARAMETER-ANALYSIS OF AGE-RELATED DEGENERATIONS OF COMPONENTS OF LUMBAR SPINE SEGMENTS IN COMPRESSION
}

Márta Kurutz ${ }^{1}$, László Oroszváry ${ }^{2}$

${ }^{1}$ Budapest University of Technology and Economics

${ }^{2}$ Knorr Bremse Hungaria Ltd

kurut2m@eik.bme.bu

\begin{abstract}
3D finite element parameter analysis of the degeneration-sensitivity of the material properties of the components of lumbar spinal motion segments is presented for physiologic compression load. The aim was to determine that material behaviour which dominantly determines the mechanical behaviour of the aging spine. Systematic numerical simulation was executed by using a finite element model validated by experimental results for both tension and compression. It was concluded that to keep the hydrostatic compression state of nucleus is the most important factor to maintain the stability of lumbar segments in the first period of aging process.
\end{abstract}

Keywords: finite element parameter analysis, lumbar motion segments, compression, material property, degeneration

\section{Introduction}

Using numerical simulation we can find out how the stability of spinal motion segments is influenced by the healthy or degenerated state of each of its components, or rather the healthy or degenerated state of which of its components has the dominant effect on the degeneration or failure of the whole segment. At the same time, by numerical parameter-analysis we can determine that components the material moduli of which needs the most care and correctness to be collected for numerical analyses of spinal degeneration. In this study a systematic parameteranalysis is presented to determine the separated effect of each material parameter of the components of the lumbar spine segments on the mechanical behaviour and stability of the whole segment.

Degeneration represents special and injurious changes in the structure, composition and function of spine or part of the spine, caused by aging or by certain environmental external effects. ${ }^{1}$ The age-related changes of spine start generally in the intervertebral discs; within the disc nucleus, altering markedly the mechanics of load transfer and spinal stability. ${ }^{2}$ The age-related changes of the disc are manifested in loss of hydration, a drying and stiffening procedure in the texture of both nucleus and annulus. ${ }^{3}$ Aging procedures are often accompanied by other kinds of degeneration: buckling, lesions, tears, fiber break in the annulus or osteoporotic changes in the vertebral bone.

The goal of this study was to obtain numerical conclusions for the separated mechanical effects of the material moduli of each components of the lumbar motion segment on the degeneration of the whole segment. 


\section{Methods}

For the numerical parameter-analysis, the finite element model of a typical lumbar motion segment was used that we developed earlier in ANSYS system, ${ }^{4}$ representing five degeneration grade of aging. A 3D model of a typical lumbar segment L4-5 was created by using Pro/Engineer code. The geometry and tissue volumes were obtained by the anatomical measures of a typical lumbar segment. ${ }^{5,6}$ Cortical and cancellous bone of vertebrae was separately modeled, including posterior bony elements and facet joints. The height of the disc was considered to decrease linearly from 10 to $6 \mathrm{~mm}$ for the five aging degeneration degrees. The endplates were divided into external bony and central cartilaginous part. Accordingly, the annulus matrix was divided into internal and external ring and three layers of annulus fibers. ${ }^{7}$ The FE mesh was generated by ANSYS Workbench and the connections between the several geometrical components were integrated to the FE model by ANSYS Classic. The FE model consisted of solid, shell and bar elements. Annulus matrix, nucleus, cancellous bone, articular joints and different types of attachments were modeled by solid elements; cortical shells and endplates were modeled by shell elements. The compatibility between the adjacent solid and shell elements was guaranteed by contact elements. All ligaments were modeled by shell elements.

Table 1 shows the material moduli of healthy lumbar motion segment. For the bony elements and endplates, for both tension and compression, linear elastic isotropic materials were applied, based on the literature. Annulus ground substance and nucleus were considered linear elastic for compression and bilinear elastic for tension. Nucleus and annulus matrix were considered linear elastic. Collagen fibers of the annulus were considered as bilinear elastic isotropic tension-only material. All the seven ligaments were integrated in the model with bilinear elastic tension-only materials of the literature again.

\begin{tabular}{|l|c|c|}
\hline \multicolumn{1}{|c|}{ Components of FSU } & $\begin{array}{c}\text { Young's mod } \\
{[\mathrm{MPa}]}\end{array}$ & $\begin{array}{c}\text { Poisson's } \\
\text { ratio }\end{array}$ \\
\hline vertebral cortical bone & 12000 & 0,3 \\
\hline posterior elements, facet & 3500 & 0,3 \\
\hline vertebral cancellous bone & 150 & 0,3 \\
\hline bony endplate & 12000 & 0,3 \\
\hline cartilaginous endplate & 100 & 0,4 \\
\hline nucleus & 1 & 0,499 \\
\hline annulus ground substance, internal & 4 & 0,45 \\
\hline annulus ground substance, external & 5 & 0,40 \\
\hline annulus fibers & $500 / 400 / 300^{*}$ & - \\
\hline anterior longitudinal ligament & $8^{* *}$ & 0,35 \\
\hline posterior longitudinal ligament & $10^{* *}$ & 0,35 \\
\hline other ligaments & $5^{* *}$ & 0,35 \\
\hline \multicolumn{2}{|c|}{$*$ external/middle/internal fibers, } & $* *$ tension only \\
\hline
\end{tabular}

Table 1. Material moduli of the components of healthy motion segment

Five grades of age-related degeneration process were introduced, seen in Table 2. Age-related normal degeneration processes of segment start generally in the nucleus. A healthy young nucleus is in hydrostatic compression state. During aging, the nucleus loses its incompressibility by changing gradually from fluid-like to solid material of increasing stiffness. ${ }^{8,9}$ These kinds of 
nucleus changes were modeled by decreasing Poisson's ratio with increasing Young's modulus, seen in Table 2. This behavior is generally accompanied by the stiffening process of the annulus. This procedure was modeled also by increasing Young's modulus with slightly decreasing Poisson's ratio, by distinguishing the internal capsular and the external ligamentous part of annulus, by considering the internal annulus to be weaker. The elastic moduli of annulus fibers were decreased during aging.

\begin{tabular}{|l|c|c|c|c|c|}
\hline $\begin{array}{c}\text { Grades of aging degeneration* } \\
\text { (Young's mod/Poisson's ratio) }\end{array}$ & $\begin{array}{c}\text { grade 1 } \\
\text { (healthy) }\end{array}$ & grade 2 & grade 3 & grade 4 & $\begin{array}{c}\text { grade 5 } \\
\text { (fully deg.) }\end{array}$ \\
\hline disc height [mm] & 10 & 9 & 8 & 7 & 6 \\
\hline nucleus & $1 / 0.499$ & $2 / 0.475$ & $6 / 0.45$ & $16 / 0.425$ & $36 / 0.4$ \\
\hline annulus matrix, internal ring & $4 / 0.45$ & $4,5 / 0.44$ & $6,5 / 0.43$ & $11,5 / 0.42$ & $20 / 0.41$ \\
\hline annulus matrix, external ring & $5 / 0.40$ & $6 / 0.39$ & $9 / 0.38$ & $17 / 0.37$ & $29 / 0.36$ \\
\hline cancellous bone & $150 / 0.3$ & $125 / 0.3$ & $100 / 0.3$ & $75 / 0.3$ & $50 / 0.3$ \\
\hline cartilaginous endplate & $100 / 0.4$ & $80 / 0.4$ & $60 / 0.4$ & $40 / 0.4$ & $20 / 0.4$ \\
\hline annulus fibers (ext/middle/int) & $500 / 400 / 300$ & $250 / 200 / 150$ & $125 / 100 / 75$ & $63 / 50 / 38$ & $10 / 10 / 10$ \\
\hline
\end{tabular}

Table 2. Modeling of normal age-related changes of lumbar motion segment

In the parameter-analysis we assumed that all components of the motion segment are under normal degeneration according to the data of Table 2 except for only one component that remains intact with the data of grade 1 , with no degeneration. Then, the obtained mechanical results were compared to the case where all components are normally aging and degenerating. We have chosen this method where all components are normally aging only on does not, instead of the inverse unrealistic process where only one component would aging and all the others remain young. The reason was to follow a physiologically normal degeneration procedure to determine the component the healthy state of which would have a dominant effect on the stability of the whole motion segment.

The model was validated for both compression and tension. The compression behaviour was compared to the experimental results of Adams et al. ${ }^{3}$ obtained by stress profilometry for $2000 \mathrm{~N}$ axial compressive load with endplate damage. For axial tension, the calculated elongations of discs were compared with in vivo measured elongations of lumbar FSU model L3-S1 in weightbath hydrotraction treatment. ${ }^{10,11}$

$1000 \mathrm{~N}$ axial physiologic compression load was applied for all degeneration grades for all numerical simulations. The compression load was distributed along the superior and inferior surface of the upper and lower vertebra of FSU, by applying rigid load distributor plates at both surfaces.

\section{Results}

Figures 14 illustrate the stresses, strains and stiffnesses of the different parts of the disc for the age-related degeneration process when all components of the lumbar motion segment were under aging degenerated state characterized by the material moduli seen in Table 2 except for the 
nucleus, namely, its Young's modulus, its Poisson's ratio and both of them were separately kept on their initial healthy values of grade 1.

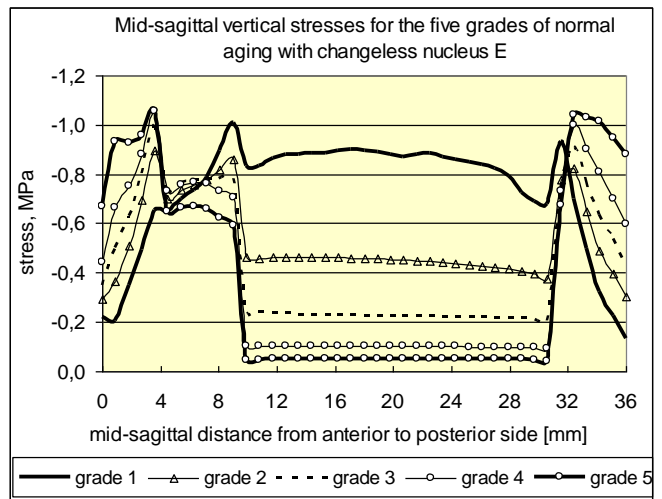

a)

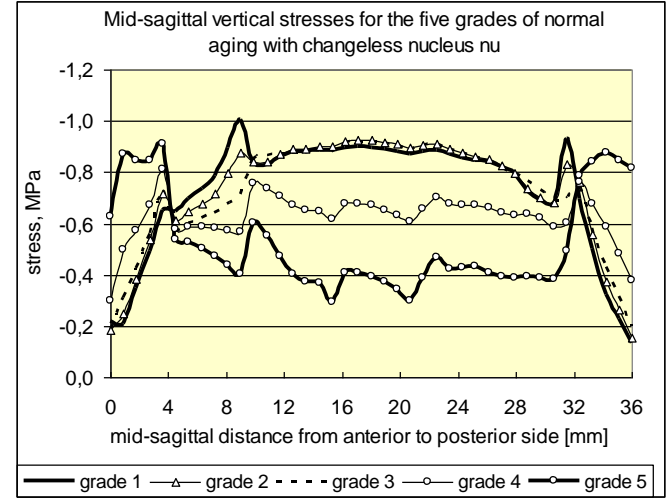

b)

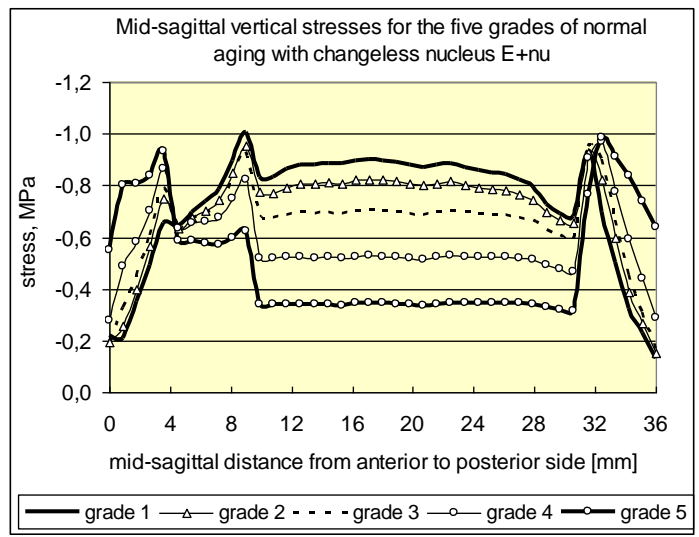

c)

Figure 1. Mid-sagittal vertical stress distribution in the disc in the five grades of aging process while a) nucleus E, b) nucleus nu and c) nucleus E+ nu keep their initial healthy values changeless

Figure 1 shows the mid-sagittal vertical compressive stress distribution in the disc from the anterior to the posterior end for the five aging degeneration grade. In Figure 1a the Young's modulus of nucleus kept its initial young value, in Figure $1 b$ its Poisson's ratio remained changeless, while in Figure $1 c$ both of them were changeless.

Figures $2 a, 2 b$ and $2 c$ show the related change of mean mid-sagittal vertical compressive stresses in the disc nucleus, in the internal and external annulus, while Figure $2 d$ shows the related posterior disc bulging during the five degeneration grade of the aging process. In Figures $3 a$ and $3 b$ the change of the internal and external compressive strains of disc can be seen, respectively. In Figures $4 a, 4 b$ and $4 c$ the vertical compressive stiffness of the nucleus, internal and external annulus can be seen, respectively, while in Figure $4 d$ the stiffness of the whole disc is illustrated. 


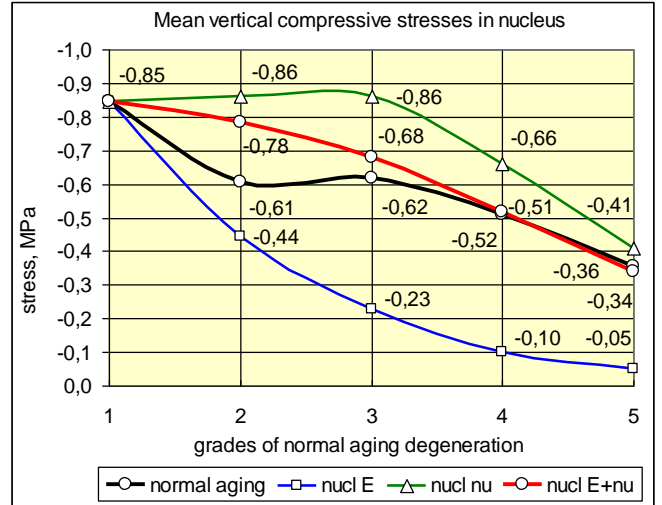

a)

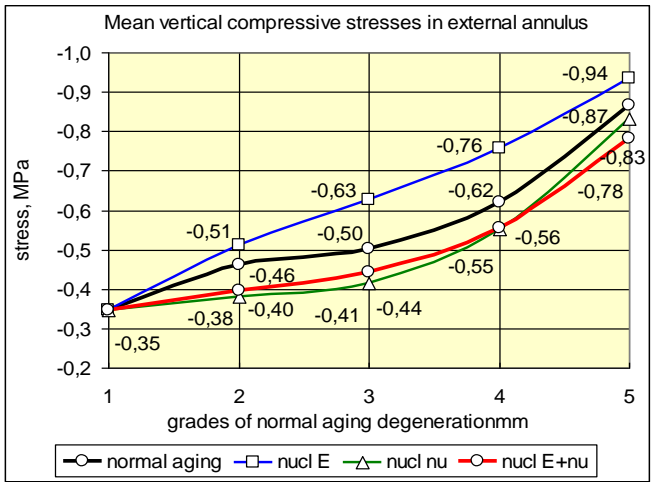

c)

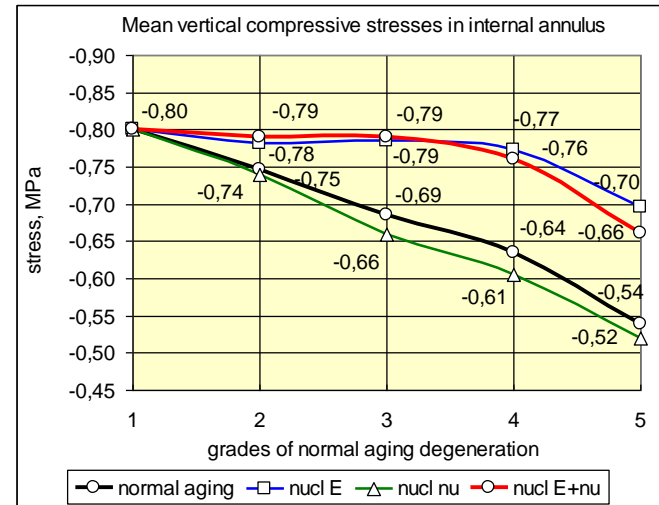

b)

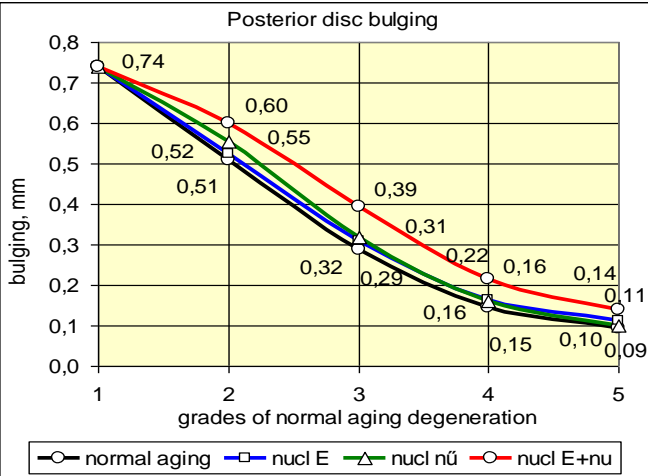

d)

Figure 2. Mean vertical compressive stresses in a) nucleus, b) internal annulus, c) external annulus and d) posterior bulging for the cases of aging degeneration process while nucleus E, nu and E+ nu keep their initial healthy values changeless

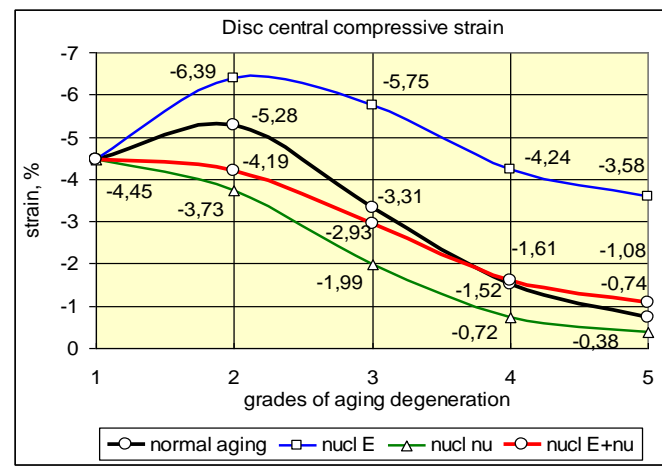

a)

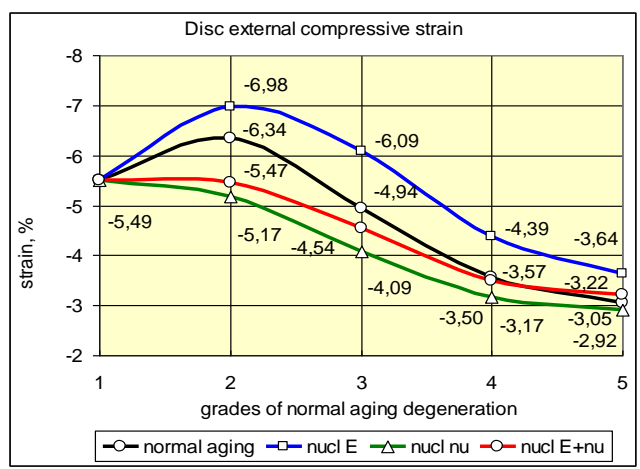

b)

Figure 3: a) Central and b) external vertical compressive strains of disc for the cases of aging degeneration process if the nucleus $\mathrm{E}$, nu and $\mathrm{E}+$ nu keep their initial healthy values changeless

When the Young's modulus of nucleus was kept separately on its initial small value $(\mathrm{E}=1 \mathrm{MPa})$ during the aging of all other components (Table 2), the stresses in nucleus rapidly decreased (by $27 \%$, in grade 2 , by $63 \%$ in grade 3, Figure 2a), in the internal and external annulus rapidly increased (by $5-11 \%$ and $15-25 \%$ in grade 2 and 3, resp., Figures $2 b$ and $2 c$ ). At the same time the central deformations in disc nucleus rapidly increased (by $21 \%$ in grade 2 and by $74 \%$ in grade 3 , 
Figure 3a), and the external deformations in annulus also increased (by $10 \%$ in grade 2 and by $23 \%$ in grade 3, Figure 3b), together with the increased posterior bulging (by $3 \%$ in grade 2 and by $7 \%$ in grade 3, Figure $2 d$ ). The most dangerous was the complete stiffness loss of nucleus (by $40 \%$ in grade 2 and by $79 \%$ in grade 3, Figure $4 a$ ) and the rapid stiffness loss of internal annulus (by $9 \%$ in grade 2 and by $20 \%$ in grade 3 , Figure $4 b$ ) that leaded to the radical stiffness decrease of the whole disc (by $15 \%$ in grade 2 and by $33 \%$ in grade 3 , Figure $4 d$ ).

Figure $1 b$ illustrates that keeping the Poisson's ratio of nucleus on the fluid-like level $(\mathrm{nu}=0,499)$ with increasing solid-like Young's modulus of it was meaningless in grade 4 and grade 5 (Table 2), yielding chaotic zigzag lines on Figure 1b. However, with small Young's modulus, in grade $2(\mathrm{E}=2$ $\mathrm{MPa})$ and $3(\mathrm{E}=6 \mathrm{MPa})$ it means that the incompressibility of nucleus is kept, that increased the stresses in nucleus (by $40-42 \%$ in grade 2 and 3, Figure 2a), and unloaded the external annulus (by $17-18 \%$ in grade 2 and 3, Figure 2c). At the same time the central deformations of disc decreased (by 30 and 40\% in grade 2 and 3, Figure 3a), and the external deformations also decreased (by 17$18 \%$ in grade 2 and 3, Figure 3b), with decrease of posterior bulging (by 9-10\% in grade 2 and 3, Figure $2 d$ ). There was a rapid stiffness increase of nucleus $(100 \%$ and $133 \%$ in grade 2 and 3, Figure $4 a$ ) and a large stiffness increase of internal annulus (29-31\% in grade 2 and 3, Figure 4b) that leaded to the radical stiffness increase of the whole disc (by $41 \%$ and $54 \%$ in grade 2 and 3 , Figure 4d).

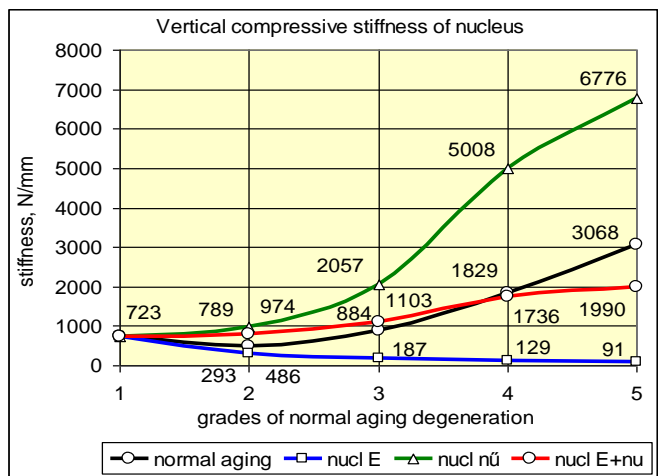

a)

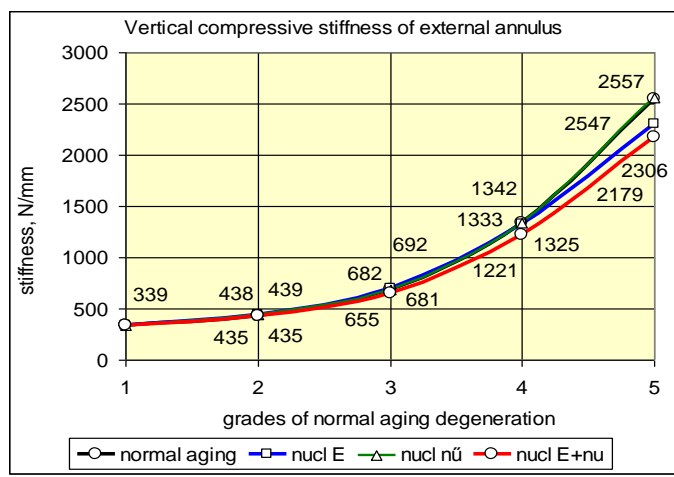

c)

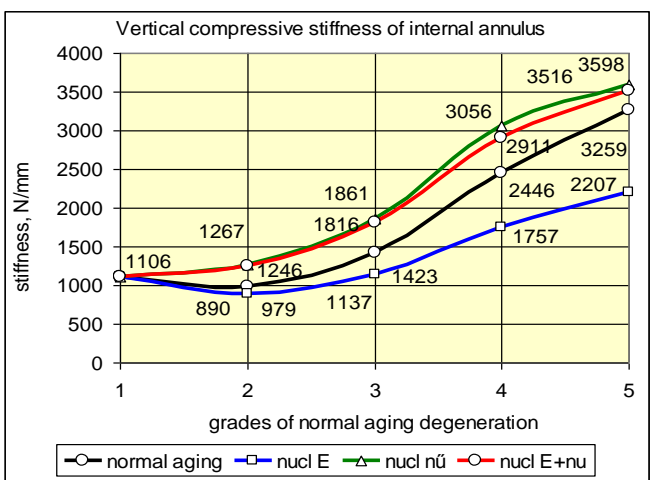

b)

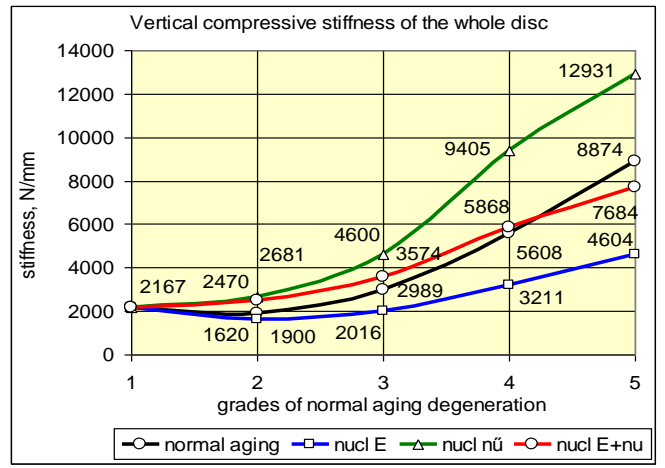

d)

Figure 4. Vertical compressive stiffness of a) nucleus, b) internal and c) external annulus and d) the whole disc for the cases of aging degeneration process if the nucleus $\mathrm{E}$, nu and $\mathrm{E}+$ nu keep their initial healthy values 
Keeping the Young's modulus and Poisson's ratio of nucleus simultaneously on its young healthy level, the most radical effect was observed in the second grade of aging degeneration process, where the stresses in nucleus increased the most (by 29\%, Figure 2a), the compressive strains in nucleus decreased the most (by 21\%, Figure 3a), similarly to the external strains (by 14\%, Figure $3 b$ ), and the stiffness of nucleus increased the most (by 62\%, Figure 4a) yielding the largest increase of the whole disc also in grade 2 (by $30 \%$, Figure $4 d$ ).

Figures 5-8 illustrate the stresses, strains and stiffnesses of the different parts of the disc for the age-related degeneration process when all components of the lumbar motion segment were under normal aging degenerated state (Table 2) except for the annulus (internal and external together), namely, its Young's modulus, its Poisson's ratio and both of them were separately kept on their initial healthy values of grade 1 .

Figures $5 a$ and $5 b$ illustrate the mid-sagittal vertical stress distribution for the five grades of aging if the annulus Young's modulus and Poisson's ratio were kept changeless. The related mean vertical compressive stresses in nucleus, in internal and external annulus are seen in Figures $6 a, 6 b$ and $6 c$, respectively, while the posterior bulging is seen in Figure $6 d$. The related central and external vertical compressive strains of the disc are illustrated in Figures $7 a$ and $7 b$, respectively. Figures $8 a$, $8 b, 8 c$ and $8 d$ show the vertical compressive stiffness of the nucleus, internal and external annulus, and the whole disc, respectively.

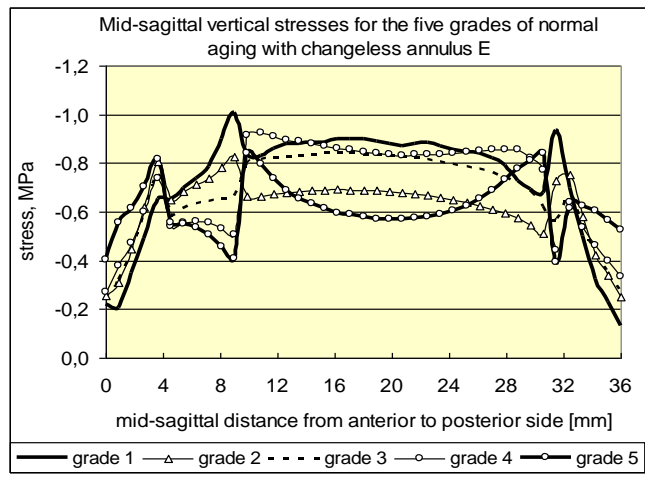

a)

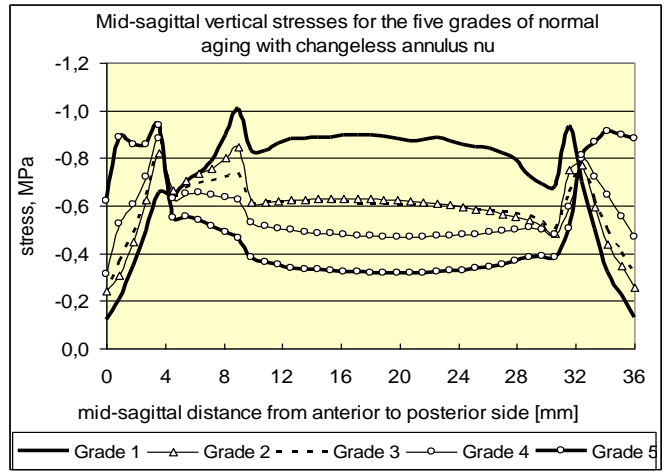

b)

Figure 5. Mid-sagittal vertical stress distribution in the five grades of aging process when a) the Young's modulus, b) the Poisson's ratio of both the internal and external annulus are separately kept changeless

If the Young's moduli of internal and external annulus were kept on their initial values $(\mathrm{E}=4 \mathrm{MPa}$ and $\mathrm{E}=5 \mathrm{MPa}$, respectively) during the gradual aging of all other components of the motion segment (Table 2), the stresses in the aging nucleus rapidly increased (by $7 \%$, in grade 2, by $29 \%$ in grade 3, and by $68 \%$ in grade 4, Figure 6 a); while both in the internal and external annulus decreased (by $8-13 \%$ in grade 3 , Figures $6 b$ and $6 c$ ). At the same time the central deformations of disc rapidly increased (by $12 \%$ in grade 2 and by $43 \%$ in grade 3, Figure $7 a$ ), and the external deformations also increased (by 10\% and 36\% in grade 2 and 3, Figure $7 b$ ) in harmony with the posterior bulging increase (14\% in grade 2 and $49 \%$ in grade 3, Figure $6 d$ ). There was a small decrease in the stiffness of nucleus $(5-10 \%$ in grade 2 and 3 , Figure $8 a)$, but both in the internal and external annulus a considerable stiffness loss was observed (11\% and $36 \%$ in grade 2 and 3, Figures $8 b$ and $8 c$ ) that leaded to the radical stiffness decrease of the whole disc (by $10 \%$ in grade 2 and by $27 \%$ in grade 3 , Figure $8 d$ ). 
It can be seen in Figures 6, 7 and 8 that the Poisson's ratio of the internal and external annulus in itself has no significant effect on the stresses and deformations of the disc or on the stiffness of the disc components and the whole disc. If keeping simultaneously the Young's modulus and Poisson's ratio of internal and external annulus on their young healthy level, the effect was similar to the separated influence of Young's modulus of annulus in Figures 6, 7 and 8.

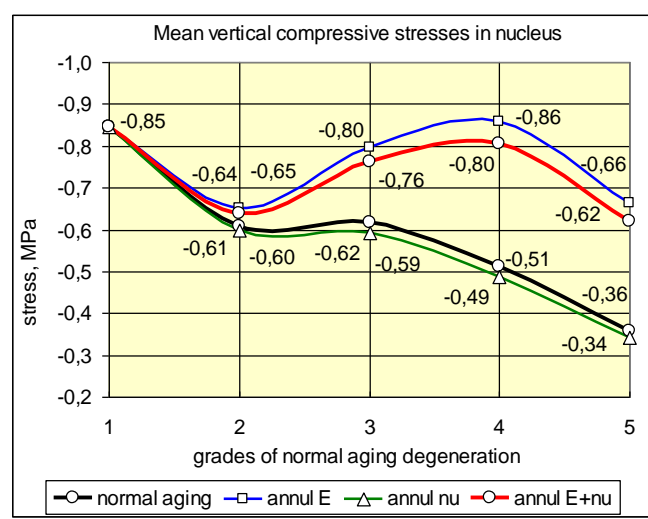

a)

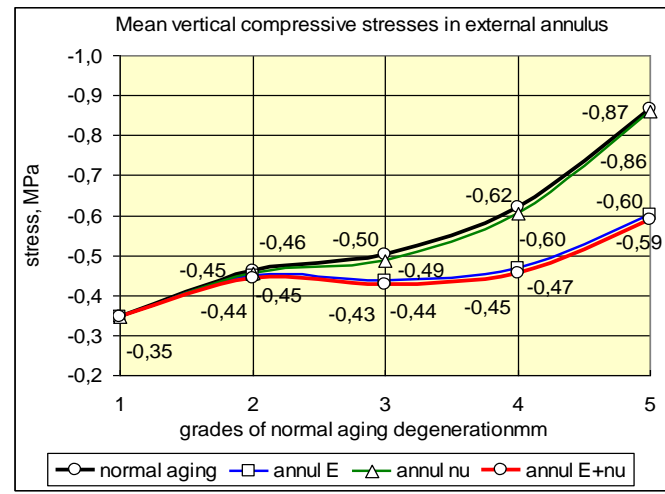

c)

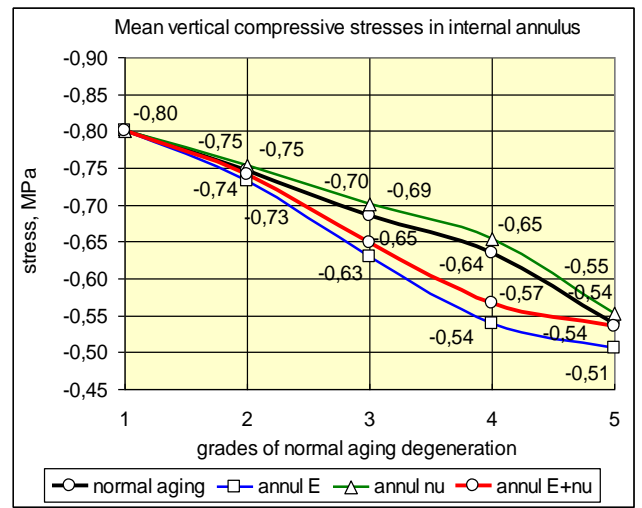

b)

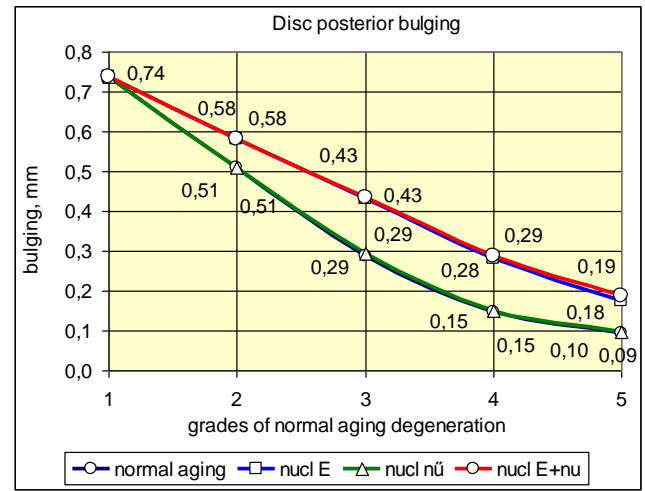

d)

Figure 6. Mean vertical compressive stresses in a) nucleus, b) internal annulus, c) external annulus and d) posterior bulging for the cases of aging degeneration process when $\mathrm{E}$, nu and $\mathrm{E}+$ nu of both the internal and external annulus are kept on their initial healthy values changeless

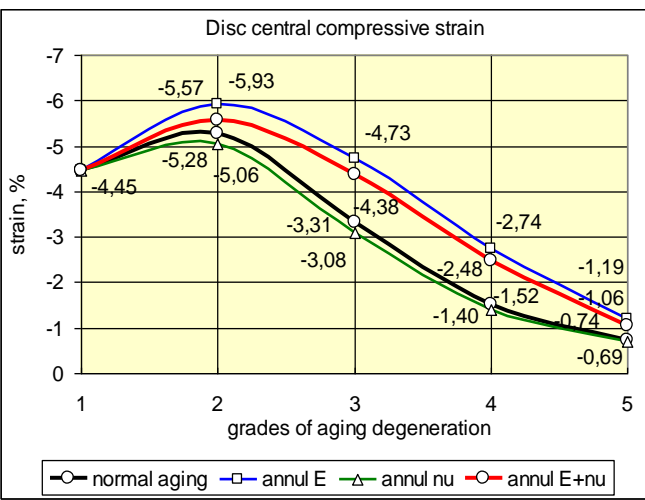

a)

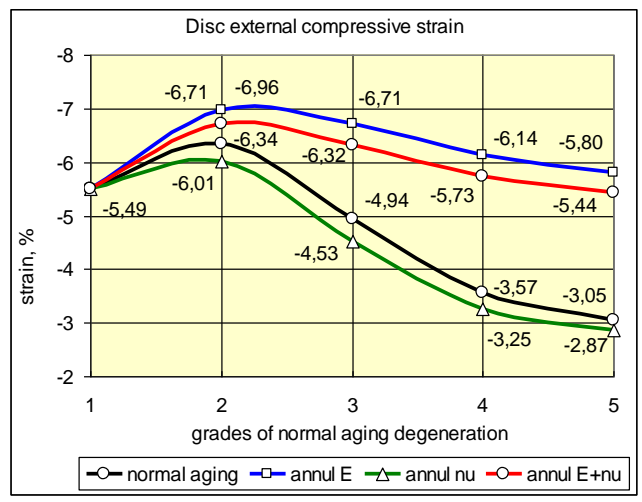

b)

Figure 7. a) Central and b) external vertical compressive strains of disc in aging degeneration process if $E$, nu and $\mathrm{E}+$ nu of both the internal and external annulus are kept on their initial healthy values 


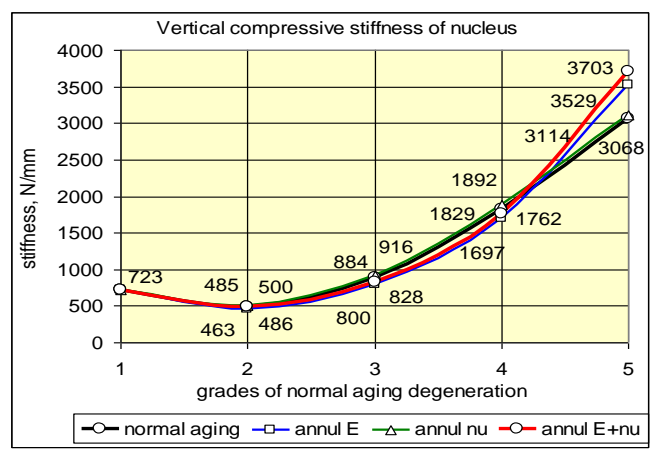

a)

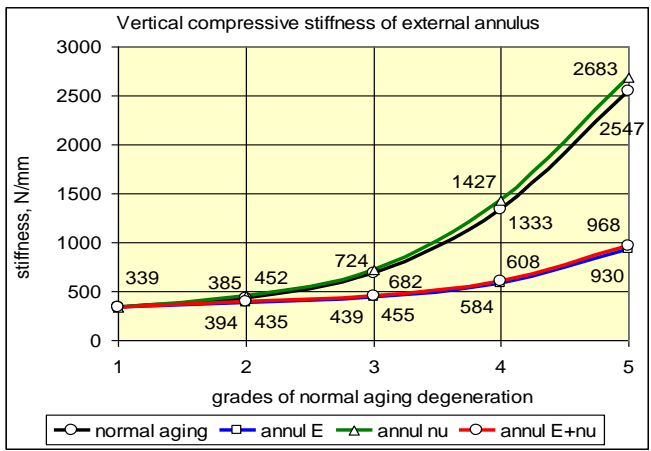

c)

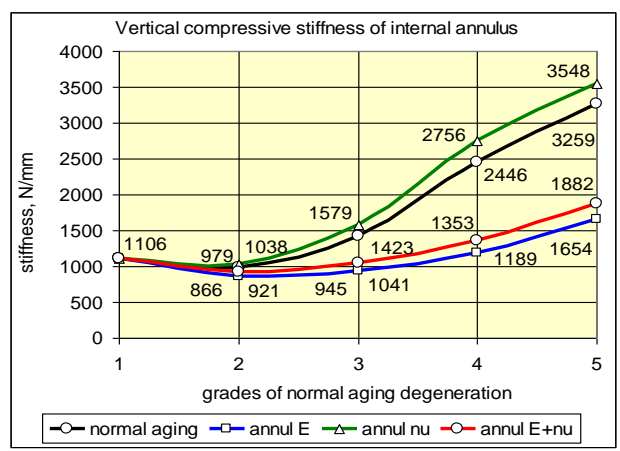

b)

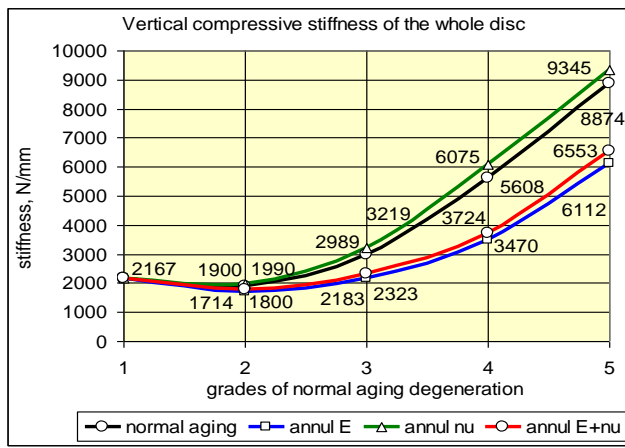

d)

Figure 8. Vertical compressive stiffness of a) nucleus, b) internal and c) external annulus and d) the whole disc for the cases of aging degeneration process if $\mathrm{E}$, nu and $\mathrm{E}+$ nu of both the internal and external annulus are kept on their initial healthy values changeless

Figures 9-11 illustrate the stresses, strains and stiffnesses of the different parts of the disc for the age-related degeneration process when all components of the lumbar motion segment were under normal aging degenerated state (Table 2) except for the vertebral cancellous bone and the cartilaginous endplate, when the Young's modulus of them were separately and together kept on their initial healthy values of grade 1.

In Figures $9 a, 9 b$ and $9 c$ the change of the mean vertical compressive stresses in nucleus, internal and external annulus can be seen, respectively, while the posterior bulging is seen in Figure $9 d$ during the five grades of aging degeneration. The related central and external vertical compressive strains of the disc are illustrated in Figures $10 a$ and $10 b$, respectively. Figures $11 a, 11 b$ and $11 c$ show the vertical compressive stiffness of the nucleus, the internal and external annulus, respectively, while in Figure $11 d$ the stiffness of the whole disc can be seen for the grades of aging degeneration process. 


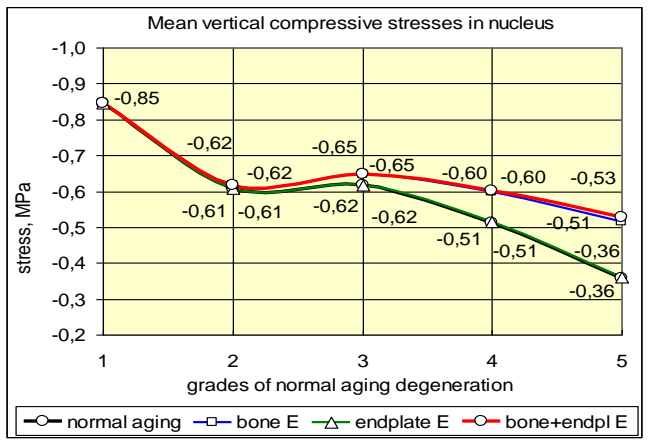

a)

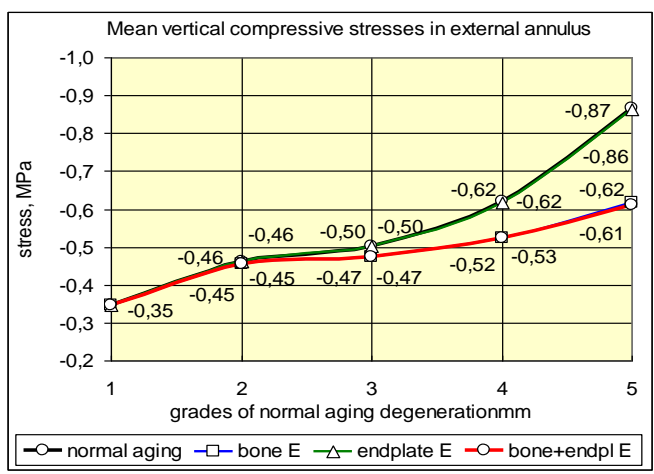

c)

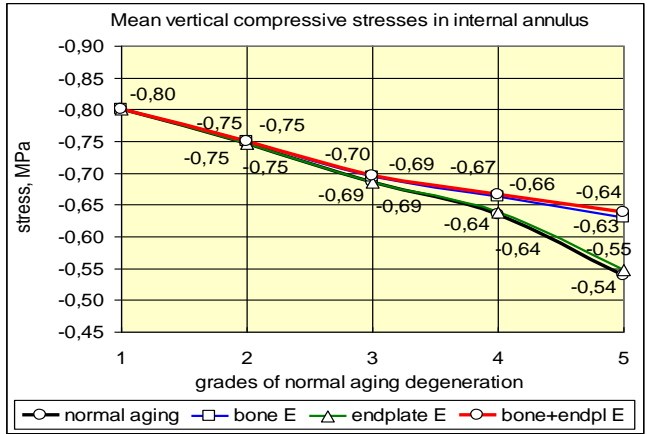

b)

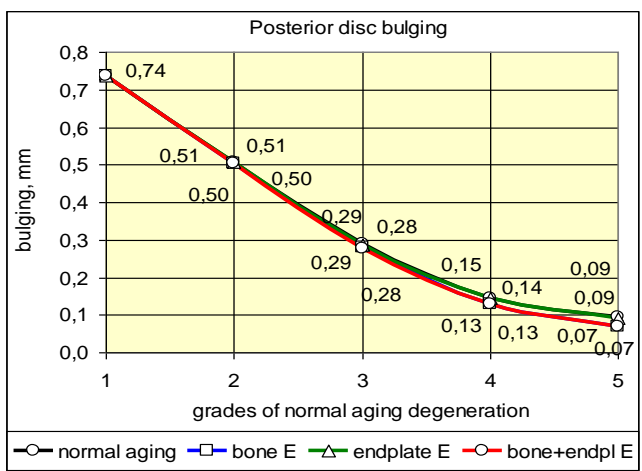

d)

Figure 9. Mean vertical compressive stresses in a) nucleus, b) internal annulus and c) external annulus for the cases of aging degeneration process while the vertebral cancellous bone E, cartilaginous endplate $\mathrm{E}$ and bone + endplate E keep their initial healthy values changeless

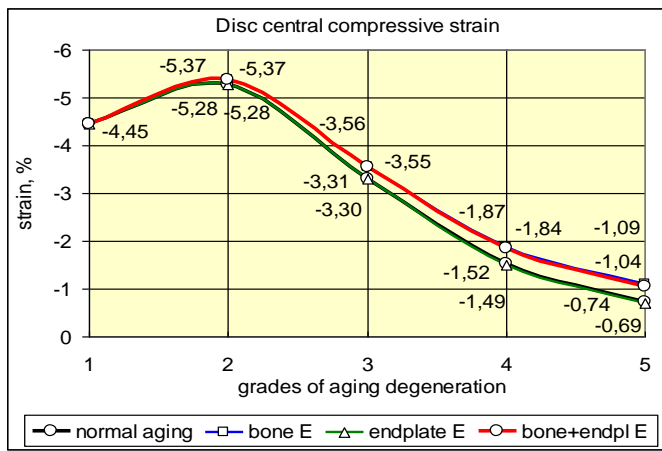

a)

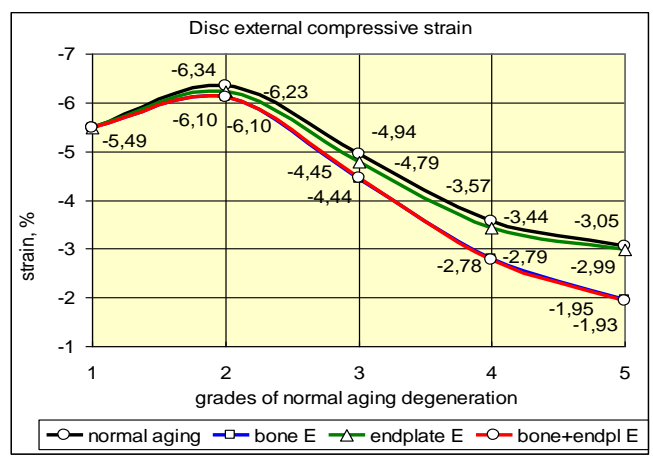

b)

Figure 10. a) Central and b) external vertical compressive strains of disc for the cases of aging degeneration process if the vertebral cancellous bone E, cartilaginous endplate E and bone + endplate E keep their initial healthy values changeless

If the Young's modulus of the vertebral cancellous bone was kept on its original strong value $(\mathrm{E}=150 \mathrm{MPa})$ while all other components were under gradual aging (Table 2), in the degeneration grade 2, 3 and 4 the stresses in the nucleus slightly increased (by 1\%, 5\%, 17\%, Figure $9 a$ ), in the internal annulus also increased (by $0 \%, 1 \%, 4 \%$, Figure 9b), while in the external annulus decreased 
(by $1 \%, 5 \%, 15 \%$, Figure $9 c$ ). At the same time the central deformations of disc increased (by $2 \%$, $7 \%, 23 \%$, Figure 10a), and the external deformations decreased (by 4\%, 10\%, 22\%, Figure 10b) in harmony with the posterior bulging that also but slightly decreased (by 1\%, 4\%, 11\%, Figure 9d) . There was a small decrease in the stiffness of nucleus $(0 \%, 2 \%, 5 \%$, Figure $11 a)$, and a small increase in the internal and external annulus $(2-3 \%, 5 \%, 8-14 \%$, Figures $11 \mathrm{~b}$ and $11 \mathrm{c})$ yielding only a small stiffness increase on the whole disc $(1 \%, 3 \%, 7 \%$, Figure $11 d)$.

It can be seen in Figures 9, 10 and 11 that the Young's modulus of endplate in itself has practically no effect on the stresses and deformations of the disc or on the stiffness of the disc components or that of the whole disc. If keeping simultaneously the Young's modulus of trabecular bone and endplate on the young healthy level, the effect was similar to the separated influence of the Young's modulus of bone, seen in Figures 9, 10 and 11.

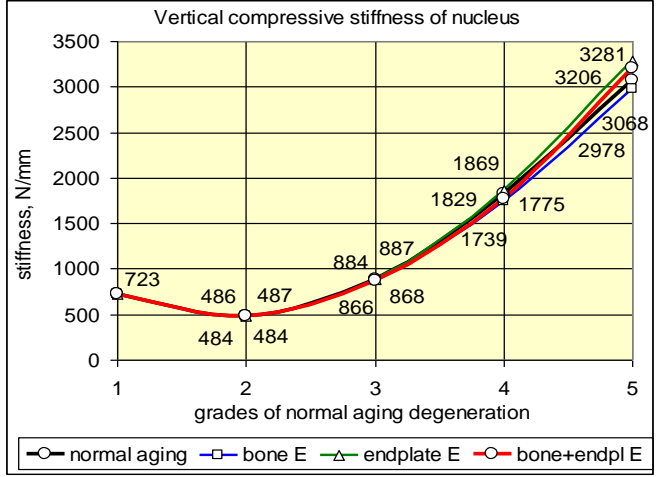

a)

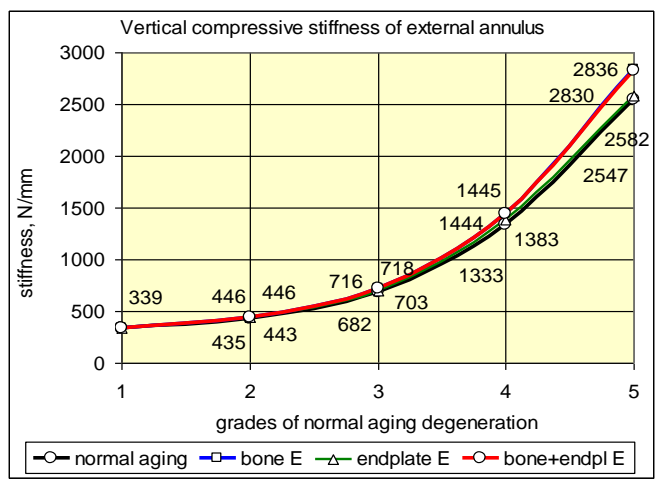

c)

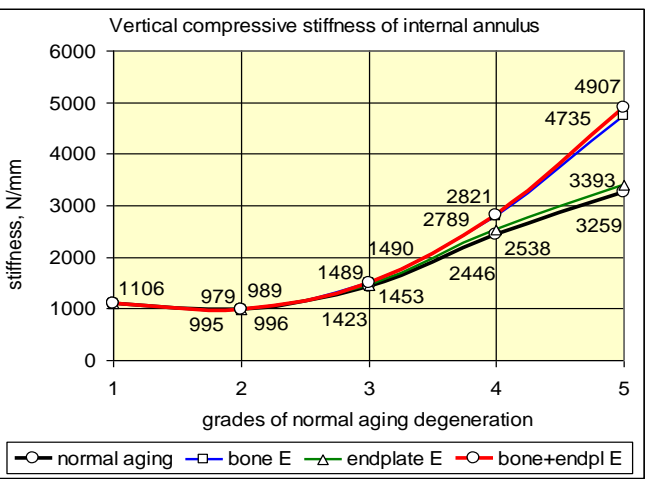

b)

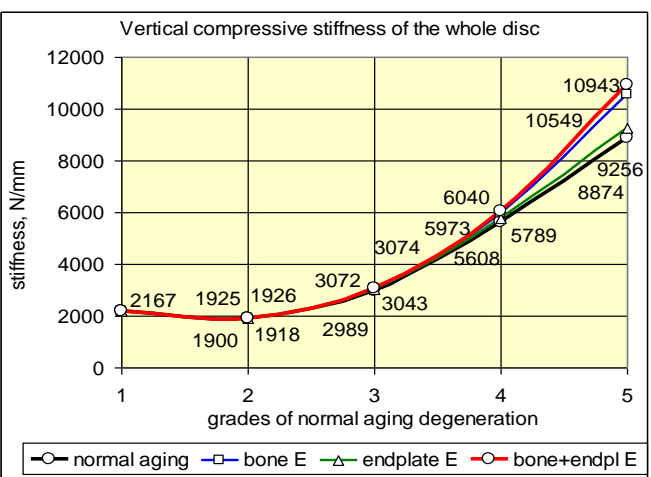

d)

Figure 11. Vertical compressive stiffness of a) nucleus, b) internal and c) external annulus and d) the whole disc for the cases of aging degeneration process if the vertebral cancellous bone $\mathrm{E}$, cartilaginous endplate $\mathrm{E}$ and bone + endplate $\mathrm{E}$ keep their initial healthy values changeless

\section{Discussion and conclusion}

Although the Young's modulus and the Poisson's ratio changes together during aging, by finite element numerical analysis the effect of them can be analyzed separately. It can be concluded that in the material modeling of spinal segments for numerical simulations, the Young's modulus and 
Poisson's ratio of nucleus need special care since the mechanical behaviour of the components and the whole segment is very sensitive to these data.

It was demonstrated that a soft nucleus with lost hydrostatic state is very dangerous yielding the complete loss of load bearing capacity of nucleus. This happens when the young nucleus quasi burst out due to some traumatic or other accidental effect yielding extreme shortening and complete stiffness loss in nucleus (Figures $3 a$ and $4 a$ ) and a large stiffness lost in the whole disc (Figure $4 d$ ) leading to the rapid overload and injury of the internal and external annulus (Figures $2 b$ and 2c). Consequently, the most important is to keep the hydrostatic stress state in the young nucleus about the second degeneration degree, in the young adult age. Keeping the hydrostatic stress state in this age can increase the compression load bearing capacity of nucleus by $30-40 \%$ and the stiffness of the whole disc by $30 \%$. The importance of keeping the incompressibility of nucleus in the first period of aging is supported by several authors ${ }^{3,12,13}$ stating that light degeneration of disc leaded to instability, while the stability restored with further degeneration, since lightly degenerated discs had the smallest stiffness. Since the stiffness of young disc is influenced mainly by the state of nucleus, it is obvious that the effect of loss of hydrostatics is crucial in the starting period of aging process.

The Poisson's ratio of annulus in itself has very small effect on the mechanics of disc, however, the effect of Young's modulus of annulus in itself and together with the Poisson's ratio is very important (Figures 6,7 and 8). The stiffening of annulus during normal aging is useful, since it helps to maintain the stability of disc. Indeed, ${ }^{3,12,13}$ concluded that after the instability in younger age the stability restored with further degeneration, since the stiffness increased during further degeneration.

The stiffness of the cartilaginous endplate in itself has also a very small influence, since it is supported by the vertebral cancellous bone, thus, the effect of it depends on the state of the bone (Figures. 9, 10 and 11). We can say that the effect of the bone is important mainly for the elderly. In the first half of the aging process, the strong bone has a small effect equally on the stresses, deformations or stiffness. In the elderly, in grade 4 and 5, the strong bone increases the load transfer through the nucleus, and unloads the external annulus, it increases the central and decreases the external shortenings of disc by decreasing the bulging that can press the nerve roots causing load back problems (Figures 9 and 10). On the contrary, in the case of osteoporosis, the load transfer and the deformations move from inside to outside causing the overload of the external annulus, leading to strong bulging and injurious degeneration. However, the stiffness of disc is slightly influenced by the state of the bone.

By the separated analysis of the material moduli it can be concluded that to keep the hydrostatic stress state in the nucleus is crucial important in the first period of aging process. The loss of incompressibility of nucleus leads to the loss of load bearing capacity of nucleus and the injurious overload of the internal and external annulus. Osteoporosis moves the load transfer from the central to the external region that can lead to the breakdown of the cortical bone of vertebrae.

\section{REFERENCES}

1. Adams MA, Freeman BJ, Morrison HP, Nelson IW, Dolan P. Mechanical initiation of intervertebral disc degeneration, Spine, 2000;25(13):1625-36. 
2. Ferguson SJ, Steffen T. Biomechanics of aging spine, Eur. Spine J., 2003;12(Suppl.2):97-103.

3. Adams MA, Bogduk N, Burton K, Dolan P. The Biomechanics of Back Pain, Churchill Livingstone, Edinburgh, London, New York, Oxford, Philadelphia, 2002. p. 238p.

4. Kurutz M, Oroszváry L. Finite element analysis of weightbath hydrotraction treatment of degenerated lumbar spine segments in elastic phase, Journal of Biomechanics, 2010;43(3):433-41.

5. Denoziere G. Numerical modeling of ligamentous lumbar motion segment, Master thesis, Georgia Institute of Technology, 2004.

6. Panjabi MM, Oxland T, Takata K, Goel V, Duranceau J, Krag M. Articular facets of the human spine, quantitative three dimensional anatomy, Spine, 1993;18(10):1298-1310.

7. Kurutz M, Oroszváry L. Finite Element Analysis of Long-Time Aging and Sudden Accidental Degeneration of Lumbar Spine Segments in Compression, Biomechanica Hungarica, 2013;VI(1):22334.

8. Iatridis JC, Wedenbaum M, Setton LA, Mow VC. Is the nucleus pulposus a solid or a fluid? Mechanical behaviours of the nucleus pulposus of the human intervertebral disc, Spine, 1996;21(10):1174-84.

9. Iatridis JC, Setton LA, Wedenbaum M, Mow VC. Alterations in the mechanical behavior of the human lumbar nucleus pulposus with degeneration and aging. J. of Orthopaedic Research, 1997;15(2):318-22.

10. Kurutz M, Bender T. Weightbath hydrotraction treatment - application, biomechanics and clinical effects, J. of Multidisciplinary Healthcare, 2010;(3):19-27.

11. Kurutz M, Bene É, Lovas A. In vivo deformability of human lumbar spine segments in pure centric tension, measured during traction bath therapy, Acta of Bioengineering and Biomechanics, 2003;5(1):67-92.

12.Schmidt H, Kettler A, Rohlmann A, Claes L, Wilke HJ. The risk of disc prolapses with complex loading in different degrees of disc degeneration - a finite element analysis, Clinical Biomechanics, 2007;22(9):988-98.

13. Tang XJ, Chen QX, Liu YS, Li FC. Analysis of lumbar disc degeneration using three-dimensional nonlinear finite element method, (Article in Chinese), Zhonghua Yi Xue Za Zhi, 2008;88(23):1634-8.

The authors gratefully acknowledge the Hungarian Scientific Research Fund OTKA for providing financial support in the frame of the grant $K-075018$. 